\title{
Consulta de enfermagem a pacientes com fissuras labiopalatais
}

\author{
Nursing consultation for patients with cleft lip and palate \\ Consulta de enfermería para pacientes con labio leporino y paladar hendido
}

Maria Julia Navarro Kassim ${ }^{1 *}$, Fabiana Gonçalves de Oliveira Azevedo Matos ${ }^{1}$, Marcielle Cândido², Gabriele da Silva Borges ${ }^{1}$, Luciana Paula Grégio D Arce Rodrigues'.

\section{RESUMO}

Objetivo: Relatar a experiência vivenciada, enquanto residentes de enfermagem, durante a consulta de enfermagem a pacientes com fissuras labiopalatais, durante os meses de março de 2020 a março de 2021 em um centro de referência no sul do país. Relato de experiência: Após a coleta de dados, eram elencados os diagnósticos prioritários e realizado o planejamento e a prescrição dos cuidados de enfermagem para a obtenção dos resultados de saúde desejados. Dentre os achados, os aspectos que mais se destacaram durante as consultas de enfermagem eram relacionados à alimentação e ao vínculo familiar do paciente acometido. A consulta de enfermagem sistematizada, com foco na gestão do cuidado, orienta o paciente e sua família de forma a desmistificar a sua condição, emponderando-os para o autocuidado e para o longo processo de reabilitação. Cabe ao enfermeiro o papel de acolher, orientar e propor estratégias de cuidado que favoreçam o equilíbrio e o bem-estar biopsicoespiritual dos pacientes com fissuras labiopalatais, proporcionando um cuidado qualificado, reflexivo e resolutivo. Considerações finais: A experiência com a realização da Consulta de Enfermagem permitiu a compreensão da sua importância para o processo de reabilitação dos pacientes com anomalias craniofaciais.

Palavras-chave: Enfermagem no consultório, Fissura palatina, Fenda labial, Reabilitação.

\begin{abstract}
Objective: To report the experience lived, as nursing residents, during the nursing consultation to patients with cleft lip and palate, during the months of March 2020 to March 2021 at a referral center in the south of the country. Experience report: After data collection, priority diagnoses were listed and nursing care planning and prescription was carried out to obtain the desired health results. Among the findings, the aspects that stood out the most during nursing consultations were related to food and the family bond of the affected patient. The systematized nursing consultation, focused on care management, guides the patient and his family in order to demystify their condition, empowering them for self-care and for the long process of rehabilitation. It is the nurse's role to welcome, guide and propose care strategies that favor the balance and biopsychospiritual well-being of patients with cleft lip and palate, providing qualified, reflective and resolving care. Final considerations: The experience with the Nursing Consultation allowed the understanding of its importance for the rehabilitation process of patients with craniofacial anomalies.
\end{abstract}

Key words: Office nursing, Cleft palate, Cleft lip, Rehabilitation.

\section{RESUMEN}

Objetivo: Informar la experiencia vivida, como residentes de enfermería, durante la consulta de enfermería a pacientes con labio leporino y paladar hendido, durante los meses de marzo de 2020 a marzo de 2021 en un centro de referencia en el sur del país. Informe de experiencia: Después de la recolección de datos, se 1 Universidade Estadual do Oeste do Paraná (UNIOESTE), Cascavel - PR. *E-mail: mjkassim_enfermagem@hotmail.com
2 Hospital Universitário do Oeste do Paraná (HUOP), Cascavel - PR. 
enumeraron los diagnósticos prioritarios y se realizó la planificación y prescripción de cuidados de enfermería para obtener los resultados de salud deseados. Entre los hallazgos, los aspectos que más se destacaron durante las consultas de enfermería fueron los relacionados con la alimentación y el vínculo familiar del paciente afectado. La consulta de enfermería sistematizada, enfocada a la gestión del cuidado, orienta al paciente y su familia para desmitificar su condición, empoderándolos para el autocuidado y para el largo proceso de rehabilitación. Es función del enfermero acoger, orientar y proponer estrategias de atención que favorezcan el equilibrio y el bienestar biopsicoespiritual de los pacientes con labio y paladar hendido, brindando cuidados calificados, reflexivos y resolutivos. Consideraciones finales: La experiencia con la Consulta de Enfermería permitió comprender su importancia para el proceso de rehabilitación de pacientes con anomalías craneofaciales.

Palabras clave: Enfermería de consulta, Paladar hendido, Labio leporino, Rehabilitación.

\section{INTRODUÇÃO}

A fissura labiopalatal é uma malformação anatômica que ocorre entre a $8^{\underline{a}}$ e a $12^{\underline{a}}$ semana de vida intrauterina por falha na fusão dos processos maxilares, nasal médio e/ou dos processos palatinos (ARARUNA RC e VENDRÚSCOLO DMS, 2000; MATOS FGOA, et al., 2020). No Brasil, a incidência da fissura labiopalatal é de uma para cada 650 nascidos vivos e a prevalência no mundo é de 1,53 casos para cada mil nascidos vivos (FREIRE AL et al., 2017).

De acordo com os dados do DATASUS, no Estado do Paraná, a cada 10 pacientes portadores de anomalia genética, um apresenta fissura labiopalatal, o que reforça a necessidade de estudos com essa temática (BALTAZAR MMM, et al., 2019).

A multicausalidade etiológica da referida malformação pode decorrer de fatores genéticos (podendo ou não estar associadas às síndromes), consumo de álcool, tabaco, medicamentos, drogas, exposição à radiação, poluição, agrotóxicos e de deficiências vitamínicas no primeiro trimestre da gestação (CUNHA GFM, et al., 2019).

A literatura aponta diferentes classificações das fissuras labiopalatais, entretanto, uma das mais utilizadas é a classificação de Spina V, et al. (1972) que tem o forame incisivo como referência anatômica para a denominação das fissuras. De acordo com a referida classificação, as fissuras podem ser do tipo: pré-forame unilateral incompleta; pré-forame bilateral incompleta; pré-forame unilateral completa; pré-forame completa bilateral; transforame unilateral; transforame bilateral; pós-forame completa; e pós-forame incompleta (LISBOA PK, et al., 2020).

Os pacientes com anomalias craniofaciais podem apresentar disfunções estéticas, odontológicas fonoaudiológicas e psicossociais, agravados por estigmas, discriminações e dúvidas sobre a sua capacidade intelectual e funcional (SANTOS JVN, et al., 2020).

A lei $n^{\circ} 1.172$ de 2015 ampara os pacientes com fissura labiopalatal, garantindo o tratamento multiprofissional especializado (clínico e cirúrgico) pelo Sistema Único de Saúde (SUS) (BRASIL, 2015), sendo assim, pacientes diagnosticados durante a gestação ou após o nascimento, devem ser encaminhados precocemente para os centros especializados em reabilitação de anomalias craniofaciais para avaliação e conduta (ESPINDOLA R, 2019).

Para melhorar a qualidade de vida desta clientela, é necessário engajamento multidisciplinar para modificar a estética facial, desenvolver a deglutição e a fala, otimizar a permeabilidade das vias aéreas superiores, promover a interação social, entre outros (PINHEIRO KS, et al., 2018; JUNIOR AAS e ALMEIDA CBP, 2020).

Assim como as demais especialidades, o cuidado de enfermagem corrobora para o processo de reabilitação integral do indivíduo com anomalias craniofaciais (LISBOA, et al., 2010). A assistência de enfermagem estruturada nos moldes do processo de enfermagem (PE) é fundamental para oferecer um 
cuidado qualificado ao portador de fissura labiopalatal, e para tanto, o profissional enfermeiro deve possuir habilidades práticas e conhecimento teórico para atender as demandas dessa clientela (JUNIOR AAS e ALMEIDA CBP, 2020).

A Sistematização da Assistência de Enfermagem (SAE) operacionaliza o Processo de Enfermagem (PE) a nível hospitalar e a Consulta de Enfermagem a nível ambulatorial, organizando aspectos referentes aos recursos humanos, métodos e instrumentos essenciais para a assistência de enfermagem sistematizada (COFEN, 2009). Ambos consistem em um método de trabalho que objetiva organizar de forma metódica os aspectos do cuidado de enfermagem, sendo estruturado em cinco etapas: coleta de dados, diagnóstico, planejamento, implementação e avaliação de enfermagem (SOUZA BR, et al., 2020).

A consulta de enfermagem é uma atividade privativa do enfermeiro que favorece a identificação das necessidades assistenciais, promove o delineamento das intervenções e a avaliação do cuidado prestado (SOUZA BR, et al., 2020). Neste contexto, os enfermeiros devem estar atentos para as diversas hipóteses diagnósticas que os indivíduos possam apresentar, visto que os erros diagnósticos comprometem a assistência prestada por direcionar intervenções equivocadas (HERDMAN H e KAMITSURU S, 2018). Além de nortear as ações de enfermagem ressaltando a individualidade e as especificidades de cada paciente, a consulta de enfermagem torna o cuidado mais humanizado e favorece os registros de enfermagem (SILVA AAS, et al., 2017).

É na infância que a criança desenvolve grande parte de suas potencialidades, e para acompanhar tal desenvolvimento é necessário um olhar holístico das situações que a permeiam (HANZEN IP, et al., 2019). O papel do enfermeiro durante a consulta de enfermagem, vai além da realização de procedimentos técnicos, pois objetiva o monitoramento do desenvolvimento e do crescimento infantil, promovendo ações de promoção a saúde (OLIVEIRA VC e CADETE MMM, 2006). É por meio da consulta de enfermagem que os cuidadores são orientados a oferecer um cuidado seguro às crianças (OLIVEIRA VC e CADETE MMM, 2006).

$\mathrm{Na}$ fase adulta, o desajustamento psicossocial, a insatisfação com a aparência física, com a fala, e/ou audição podem desencadear problemas na autoimagem, autoconfiança e autoestima (SANTOS JVN, et al. 2020). É por meio desta ferramenta metodológica que o enfermeiro consegue identificar tais respostas humanas e propor estratégias de cuidado assertivas para melhorar a qualidade de vida do paciente e da sua família (CRIVELARO PMS, et al., 2020).

Por entender a importância da consulta de enfermagem para a promoção de uma assistência qualificada e para a autonomia profissional do enfermeiro (SILVA DA, et al. 2020). O objetivo do estudo foi relatar a experiência vivenciada, enquanto residentes de enfermagem, durante a consulta de enfermagem a pacientes com fissuras labiopalatais.

\section{RELATO DE EXPERIÊNCIA}

Trata-se de um estudo descritivo do tipo relato de experiência, que permitiu explorar as atividades vivenciadas durante a consulta de enfermagem aos pacientes portadores de fissuras labiopalatais em um serviço ambulatorial especializado do sul do Brasil. O estudo atendeu as exigências da Resolução 466/2012, com aprovação pelo Comitê de Ética da Universidade Estadual do Oeste do Paraná, CAEE noㅡ 36452320.0.0000.0107 e parecer $n^{\circ}$ 4.250.143.

A experiência relatada é decorrente das ações de enfermagem vinculadas ao Programa de Residência Multiprofissional em Reabilitação Integral das Anomalias Craniofaciais de uma universidade pública paranaense. O Centro de Atenção e Pesquisa em Anomalias Craniofaciais (CEAPAC) é localizado na cidade de Cascavel e atende a macrorregião oeste, abrangendo 51 municípios. É um dos três centros especializados para 0 atendimento dessa clientela no estado e conta com uma equipe multiprofissional composta por médicos, enfermeiros, odontólogos, fonoaudiólogos, nutricionistas, psicólogos, biólogos, fisioterapeutas e assistentes sociais (BALTAZAR MMM, et al., 2019). Desde a sua inauguração, em fevereiro de 2013, o CEAPAC já realizou aproximadamente 35.000 atendimentos ambulatoriais com uma média mensal de 365 procedimentos nas diferentes especialidades. 
O presente relato sumariza a experiência vivenciada durante os meses de março de 2020 a março de 2021 junto ao serviço de enfermagem, composto por uma enfermeira preceptora, uma enfermeira tutora e duas técnicas de enfermagem. Eram utilizados dois instrumentos distintos para a realização da consulta de enfermagem sendo um para conduzir a consulta de pacientes pediátricos, estruturado nos moldes da teoria de Wanda Horta (disposto em Arquivo suplementar no Anexo A) (HORTA WA, 1979). E outro para conduzir a consulta de pacientes adultos, baseado nos Padrões Funcionais de Saúde de Marjory Gordon (disposto em Arquivo suplementar no Anexo B) (GORDON M, 1982).

A consulta de enfermagem era desenvolvida por um profissional enfermeiro em uma sala específica para esta finalidade. Todas as consultas conduzidas pelos residentes de enfermagem eram supervisionadas pelo enfermeiro preceptor ou tutor da residência. A avaliação de cada paciente era realizada por meio de coleta de dados no prontuário, anamnese e exame físico para obtenção de informações precisas acerca do seu quadro clínico.

Após a coleta de dados, eram identificadas as respostas dos pacientes frente aos problemas vivenciados e enunciados os diagnósticos de enfermagem prioritários. Na sequência, era realizado o planejamento, a prescrição e a implementação do plano de cuidado específico. Dentre as atividades executadas, destacavamse orientações de educação em saúde, suporte emocional, realização de procedimentos padronizados pela instituição por meio do Procedimento Assistencial Padrão - PAP (cateterização nasoenteral/vesical, aspiração traqueal, troca de curativos, administração de medicamentos, entre outros) e referenciamento quando necessário. A avaliação dos resultados obtidos através da terapêutica adotada era realizada de forma sistemática, logo após a realização do procedimento e/ou durante a consulta de retorno do paciente ao serviço que eram agendadas de acordo com o estado atual do paciente.

Os recém-nascidos que demandavam maiores cuidados de enfermagem eram avaliados semanalmente para o acompanhamento do seu estado de saúde. O ganho ponderal era uma das maiores preocupações tanto da enfermagem como de toda a equipe multiprofissional. Após essa fase, o lactente era avaliado mensalmente pela enfermagem, nas consultas de seguimento para acompanhamento quanto ao seu crescimento e desenvolvimento. As consultas multiprofissionais são realizadas em dois momentos distintos, sendo eles: acolhimento do paciente e familiares ao serviço no momento da admissão dos mesmos e orientações pré e pós-operatórias aos pacientes com cirurgias previamente agendadas (queiloplastia, palatoplastia, rinoplastia, otoplastia, etc).

Após o procedimento cirúrgico, a consulta de enfermagem é agendada semanalmente para acompanhamento da evolução do processo cirúrgico (cicatrização da ferida operatória, seguimento das orientações nutricionais, troca de curativos e orientações gerais de saúde), sendo que todos os registros decorrentes da consulta de enfermagem são documentados no prontuário eletrônico institucional denominado Sistema de Gestão em Saúde - Philips (Tasy $\left.{ }^{\circledR}\right)$.

O Nascimento de uma criança portadora de fissura labiopalatal pode desencadear um processo de enlutamento familiar devido à quebra da expectativa do filho perfeito, podendo interferir no vínculo conjugal e culminar no abandono familiar (JUNIOR AAS e ALMEIDA CBP, 2020). Entretanto, o diagnóstico precoce da malformação, ainda no período pré-natal, pode favorecer o vínculo da mãe e da família para uma melhor aceitação do bebê (VANZ AP e RIBEIRO NRR, 2011).

É frequente que os pais se sintam abalados emocionalmente ao se depararem com o diagnóstico de uma malformação craniofacial (BUSCAGLÍA L, 2006; MANZATO AL, et al., 2020; MORAES MMV, et al., 2020). Preocupados com o preconceito e com o julgamento da sociedade com relação à anomalia, os mesmos tendem a se afastar da família/comunidade como forma de proteção e com isso, podem desenvolver patologias psicológicas de difícil tratamento (BUSCAGLÍA L, 2006; MANZATO AL, et al., 2020; MORAES MMV, et al., 2020).

Por meio do vínculo estabelecido entre enfermeiro e paciente/família durante a consulta de enfermagem é possível obter informações fidedignas sobre aspectos relativos à higiene oral, alimentação, desenvolvimento neuropsicomotor, estrutura familiar e adesão ao tratamento. 
Estudos apontam que crianças com fissuras labiopalatais podem apresentar disfunções alimentares (sucção/deglutição ineficiente, regurgitamento), problemas na dicção e na audição, déficit no crescimento e no desenvolvimento físico, entre outros (SANTOS FMR e BARBOSA VT, 2017; SANTOS EAMC e OLIVEIRA TM, 2021; SOUZA BJLD, et al., 2021). Com relação aos pacientes adultos portadores de fissuras, é frequente apresentarem sentimento de baixa autoestima, insatisfação com a aparência (principalmente no gênero feminino), sentimento de fraqueza e impotência, dificuldade de inserção no mercado de trabalho e relacionamento interpessoal (SANTOS LB, 2016; GLAESER A, et al., 2018).

Nesse contexto, a consulta de enfermagem possibilita ao enfermeiro, a identificação das respostas dos pacientes frente aos processos vivenciados e permite a elaboração de um plano de cuidados de enfermagem humanizado, holístico e individualizado, sendo possível identificar as situações de saúde/doença, contribuindo para a promoção, prevenção, recuperação e reabilitação da saúde do indivíduo, família e comunidade (NERY IS, et al., 2013).

Alguns aspectos que interferem negativamente na realização da consulta de enfermagem é a dificuldade em conciliar as atividades burocráticas e assistenciais, agravadas devido a sobrecarga de trabalho, a escassez de profissionais e a falta de apoio institucional (CRIVELARO PMS, et al., 2020). A fragilidade do ensino sobre essa metodologia durante a graduação associada à falta de conhecimento prático e teórico, também dificultam a operacionalização da mesma na rotina de trabalho do enfermeiro (KAHL C et al., 2018).

A introdução de conteúdo específico sobre a Consulta de Enfermagem ainda na graduação favorece a adesão dos enfermeiros, minimizando os fatores que dificultam a implementação da consulta de enfermagem nos serviços de saúde.

A experiência relatada evidencia a importância da Consulta de Enfermagem realizada aos pacientes com anomalias craniofaciais na instituição investigada. Por meio desta, o enfermeiro atua de forma autônoma em busca de melhores resultados de saúde e maior qualidade de vida dos pacientes, cuidadores e familiares. Cabe ao enfermeiro o papel de acolher, orientar, e juntamente com o paciente, propor estratégias de cuidado que favoreçam o equilíbrio e o bem-estar biopsicoespiritual do mesmo, visto que enfermeiro empoderado, ciente das suas competências e atribuições, proporciona um cuidado qualificado, reflexivo e resolutivo.

\section{AGRADECIMENTOS E FINANCIAMENTO}

Agradecemos a toda equipe multiprofissional do Centro de Atenção e Pesquisa em Anomalias Craniofaciais que colaboram para a excelência do cuidado prestado à referida clientela.

\section{REFERÊNCIAS}

1. ARARUNA RC, VENDRÚSCOLO DMS. Alimentação da criança com fissura de lábio e/ou palato - um estudo bibliográfico. Rev.latino-am.enfermagem, 2000; 8(2).

2. BALTAZAR MMM, et al. ATENÇÃO À SAÚDE BUCAL A PACIENTES FISSURADOS LABIOPALATAIS NO ESTADO DO PARANÁ. In: Rafael Gomes Ditterich, Guilherme Fernandes Graziani, Samuel Jorge Moysés (organizadores). (Org.). Caminhos e trajetórias da saúde bucal no estado do Paraná. 1ed.Londrina: INESCO, $2019 ; 1$.

3. BRASIL. Câmara dos Deputados. Projeto de lei no 1172, de 16 de abril de 2015. Dispõe sobre a obrigatoriedade da cirurgia reparadora de lábio leporino ou fenda palatina no Sistema Único de Saúde (SUS) e nos conveniados e dá outras providências. Disponível em: https://www.camara.leg.br/proposicoesWeb/fichadetramitacao?idProposicao=1212501. Acessado em: 30 de março de 2021.

4. BUSCAGLÍA L. OS DEFICIENTES E SEUS PAIS. Rio de Janeiro: Editora Record, 2005.

5. CONSELHO FEDERAL DE ENFERMAGEM. Resolução COFEN-358/09: dispõe sobre a Sistematização da Assistência de Enfermagem e a implementação. Brasília: COFEN; 2009. Disponível em: http://www.cofen.gov.br/resoluo-cofen-3582009_4384.html. Acessado em: 30 de março de 2021.

6. CRIVELARO PMS, et al. Consulta de enfermagem: uma ferramenta de cuidado integral na atenção primária à saúde. Braz. J. of Develop., Curitiba, 2020.

7. CUNHA GFM, et al. Gestação e fissura labiopalatina do bebê: principais dúvidas das gestantes. Rev enferm UERJ, Rio de Janeiro, 2019; 27: e34127. 
8. DOS SANTOS EAMC, de OLIVEIRA TM. Conhecimentos atuais em Fissuras Labiopalatinas: uma revisão narrativa. Revista Eletrônica Acervo Saúde, 2021; 13(2): e5870.

9. ESPÍNDOLA R. O RECONHECIMENTO DA FISSURA LABIOPALATINA COMO DEFICIÊNCIA NO ORDENAMENTO JURÍDICO BRASILEIRO. UNIVERSIDADE DO SUL DE SANTA CATARINA- UNISUL, 2019.

10. FREIRE AL, et al. Atenção à pessoa com fissura labiopalatina: proposta de modelização para avaliação de centros especializados, no Brasil. Saúde em Debate, 2017; 41 (1).

11. GLAESER A, et al. Fissura labiopalatina: avaliação do impacto psicológico utilizando a Escala de Autoestima de Rosenberg. Rev. Bras. Cir. Plást, 2018:33(2):187-195.

12. GORDON M. Nursing diagnosis: process and application. St. Louis (US). McGraw-Hill; 1982.

13. HANZEN IP, et al. DIAGNÓSTICOS, INTERVENÇÕES E RESULTADOS DE ENFERMAGEM PARA SUBSIDIAR A CONSULTA DE ENFERMAGEM À CRIANÇA. Enferm. Foco, 2019; 16-21.

14. HERDMAN H, KAMITSURU S. Diagnósticos de Enfermagem da NANDA-I: Definições e Classificação - 2018/2020. 11를 ed. Porto Alegre: Artmed; 2018.

15. HORTA WA. Processo de enfermagem. São Paulo (SP): EPU; 1979.

16. JUNIOR AAS, ALMEIDA CBP. O PROCESSO DE ENFERMAGEM APLICADO AO PACIENTE COM FISSURA DE LÁBIO E/OU PALATO: REVISÃO INTEGRATIVA. Universidade do Oeste Paulista - UNOESTE, Presidente Prudente, SP, 2020.

17. KAHL C, et al. Actions and interactions in clinical nursing practice in Primary Health Care. Rev Esc Enferm USP, 2018;52: e03327.

18. LISBÔA PK, et al. Fissura Lábio-Palatal: Uma Revisão de Literatura. Revista Brasileira de Odontologia, 2010.

19. MANZATO AL, et al. Fissuras labiopalatais congênitas: uma análise dos comportamentos e enfrentamentos paternos. SALUSVITA, 2020; 39(3).

20. MATOS FGOA, et al. Epidemiological profile of labiopalatine clefts in children cared for at a reference center in Paraná. Rev. Enferm. UFSM - REUFSM, 2020: 10(28).

21. MORAES, et al. Assistência ao portador da má formação de fissura labiopalatina. Braz. J. Hea. Rev., , $2020: 3$ (1).

22. NERY IS, et al. Dificuldades para a implantação sistematização da assistência de enfermagem em maternidades. Enfermagem em Foco ,2013; 4(1): 11-14.

23. OLIVEIRA VC, CADETE MMM. A consulta de enfermagem no acompanhamento do crescimento e desenvolvimento infantil. Revista Mineira de Enfermagem, 2006.

24. PINHEIRO KS, et al. Fissuras labiopalatinas: revisão de literatura. ARCHIVES OF HEALTH INVESTIGATION, 2018.

25. SANTOS FMR, BARBOSA VT. AS ESTRATÉGIAS DO ENFERMEIRO FRENTE Á AMAMENTAÇÃO DO RECÈMNASCIDO COM FISSURA DE LÁBIO OU PALATO. IN 17ํㅡㄹ Congresso Nacional de Iniciação Cientifica CONIC/SEMESP, 2017.

26. SANTOS JVN, et al. FISSURA LABIOPALATINA: ESTUDO DO PAPEL DO PROFISSIONAL DE SAÚDE NA DIMINUIÇÃO DOS DANOS AO PACIENTE. REVISTA CIÊNCIAS E ODONTOLOGIA. RCO, 2020;4 (1):48-55.

27. SANTOS LB. AS EXPERIÊNCIAS COM A FISSURA LABIOPALATAL E OS PROCESSOS DE ESTIGMATIZAÇÃO. Dissertação (mestrado) - Instituto de Saúde Coletiva. Universidade Federal da Bahia, Salvador, 2016.

28. SILVA AAS, et al. RELATO DE EXPERIÊNCIA SISTEMATIZAÇÃO DA ASSISTÊNCIA DE ENFERMAGEM EM UMA UNIDADE PRISIONAL FUNDAMENTADA NA TEORIA DE OREM. Rev Enferm UFSM, 2017;7(4): 725-735.

29. SILVA DA, et al. Acurácia de diagnósticos de enfermagem: revisão integrativa. ENFERMAGEM BRASIL, 2020: 167175.

30. SOUZA BJLD, et al. Assistência de enfermagem para promoção do aleitamento materno de recém-nascidos portadores de fissuras orais, 2021.

31. SOUZA BR, et al. A sistematização da assistência de enfermagem desenvolvida para um caso raro de Kérion Celsi: relato de experiência. Revista Eletrônica Acervo Saúde, 2020: (51), e3505.

32. SOUZA EAN, SANTOS RPF. A POSSIBILIDADE DE AMAMENTAÇÃO DE RECÉM-NASCIDOS PORTADORES DE FENDA LABIOPALATINA: REVISÃO DA LITERATURA. Revista Brasileira de Ciências da Saúde, 2010: 8(23).

33. SPINA V, et al. Classificação das fissuras lábio-palatinas: sugestão de modificação. Rev Hosp Clin Fac Med São Paulo, 1972: 27(1):5-6.

34. VANZ AP, RIBEIRO NRR. Escutando as mães de portadores de fissuras orais. Revista da Escola de Enfermagem da USP, 2011; 45 (3): 596-602. 